\title{
Beam Test Results of the BTeV Silicon Pixel Detector
}

\author{
G. Chiodini ${ }^{\text {a }}$, J.A. Appel ${ }^{\text {a }}$, M. Artuso ${ }^{\text {e }}$, J.N. Butler ${ }^{\mathrm{a}}$, \\ G. Cardoso ${ }^{\text {a }}$, H. Cheung ${ }^{\text {a }}$, D.C. Christian ${ }^{\text {a }}$, A. Colautti ${ }^{\text {, }}$, \\ R. Coluccia ${ }^{\mathrm{d}}$, M. Di Corato ${ }^{\mathrm{d}}$, E.E. Gottschalk ${ }^{\mathrm{a}}$, B.K. Hall ${ }^{\mathrm{a}}$, \\ J. Hoff a , P. A. Kasper ${ }^{\text {a }}$, R. Kutschke ${ }^{\text {a }}$, S.W. Kwan ${ }^{\text {a }}$, \\ A. Mekkaoui ${ }^{\text {a }}$, D. Menasce ${ }^{\text {c }}$, C. Newsom ${ }^{\text {b }}$, S. Sala ${ }^{\text {c }}$, R. Yarema ${ }^{\text {a }}$, \\ J.C. Wang ${ }^{\mathrm{e}}$, and S. Zimmermann ${ }^{\mathrm{a}}$

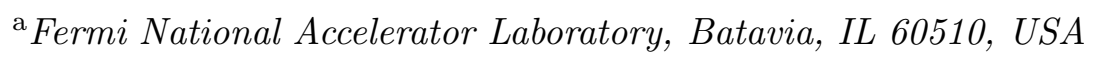 \\ ${ }^{\mathrm{b}}$ University of Iowa, Iowa City, IA 52242, USA \\ c Sezione INFN di Milano, via Celoria 16 - 20133 Milano, Italy \\ ${ }^{\mathrm{d}}$ Universitá di Milano, Dipartimento di Fisica, via Celoria 16 - 20133 Milano, Italy \\ e Syracuse University, Syracuse, NY 1344-1130, USA
}

\begin{abstract}
The results of the BTeV silicon pixel detector beam test carried out at Fermilab in 1999-2000 are reported. The pixel detector spatial resolution has been studied as a function of track inclination, sensor bias, and readout threshold.
\end{abstract}

\section{Introduction}

The BTeV collaboration has intensively beam-tested several single chip silicon pixel detector prototypes and front-end readout chips, in order to establish the basic parameters of the pixel sensors and readout chips which will be used as the building blocks of the $\mathrm{BTeV}$ vertex detector[1]. To study the pixel detector spatial resolution, a reference silicon telescope was used to project the incident beam track to the pixel sensor under test. Of particular interest was a compar- ison of the resolution obtained, using 8 bit and 2 bit charge information, for a variety of incident beam angles (from 0 to 30 degrees). Moreover, the spatial resolution was studied as a function of sensor bias and readout threshold.

\section{Experimental setup}

The tests were performed in the MTest beamline at Fermilab, with a $227 \mathrm{GeV} / \mathrm{c}$ pion beam incident on a 6 plane silicon microstrip telescope 


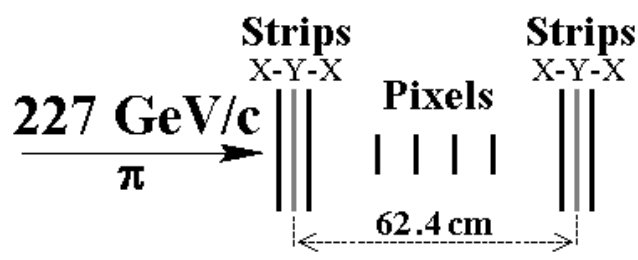

Fig. 1. Schematic drawing of the silicon telescope.

(see Figure 1), with several singlechip silicon pixel planes placed in the middle of the apparatus. The pixel sensors tested have $50 \mu \mathrm{m} \times 400 \mu \mathrm{m}$ pixel size and are all from the "first ATLAS prototype submission [2]"; up to four pixel detectors could be tested simultaneously. The silicon microstrip detectors (SSD) were read out using SVX-IIb ASIC's[3] and the data acquisition system was based on VME, adapted from the CDF SVX test stand[4]. The extrapolation accuracy of the silicon microstrip telescope at the pixel detectors location was $\sim 2.1 \mu \mathrm{m}$ for tracks with shared charge in adjacent SSD channels. The excellent spatial resolution was due to the small strip pitch $(20 \mu \mathrm{m})$ and the high pion momentum available (which minimized the multiple scattering).

The readout was triggered by the coincidence of signals from two $15 \mathrm{~cm}$ $\times 15 \mathrm{~cm}$ scintillation counters, positioned upstream and downstream of the silicon telescope and separated from each other by about $10 \mathrm{~m}$. In order to select tracks incident on the active area of the pixel detectors, the FAST_OR output signal from one of the FPIX0-instrumented pixel detectors was also required.

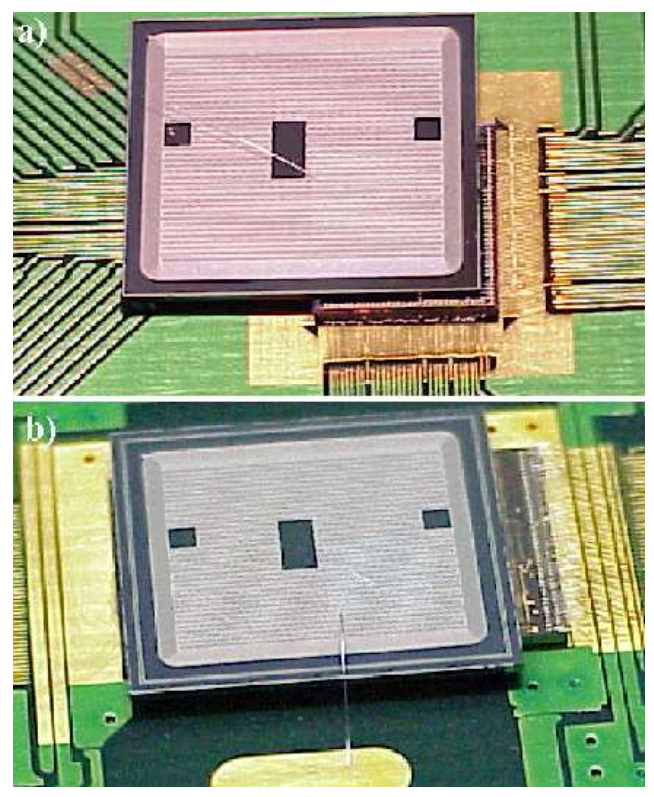

Fig. 2. FPIX0 bonded to the CiS p-stop sensor (a) and FPIX1 bonded to the Seiko p-stop sensor (b).

\subsection{FPIX0 and FPIX1 readout chips}

The FPIX0 readout chips were indium bump bonded by Boeing North America Inc. to CiS sensors (one pstop "ST1" and one p-spray "ST2"). The instrumented portion of the sensor is 11 columns $\times 64$ rows (Figure 2a). Each FPIX0 readout pixel contains an amplifier, a comparator, and a peak sensing circuit[5]. The analog output is digitized by an external 8-bit flash ADC.

The FPIX1 readout chips were indium bump bonded by Advanced Interconnect Technology Ltd to Seiko sensors (two p-stop ST1's and one pspray ST2). FPIX1 is the first implementation of a high speed readout architecture designed for $\mathrm{BTeV}$. It has 18 columns of 160 rows, and is the same size as the ATLAS single 
chip sensors (Figure 2b). However, a minor design error limited the number of rows which may be read out to $\sim 90$ per column. Each FPIX1 cell contains an amplifier, very similar to the FPIX0 amplifier, and four comparators, which form an internal 2bit flash ADC[5].

The pixel detectors were calibrated using a pulser and two x-ray sources ( $\mathrm{Tb}$ and $\mathrm{Ag}$ foils excited by an ${ }^{241} \mathrm{Am}$ $\alpha$-emitter). For most of the data taking, the discriminator threshold for the FPIX0 p-stop was set to a voltage equivalent to $2500 \pm 400 \mathrm{e}^{-}$. For the FPIX0 p-spray device the corresponding threshold was typically $2200 \pm 350 \mathrm{e}^{-}$. The amplifier noise was measured to be $105 \pm 15 \mathrm{e}^{-}$for the FPIX0 p-stop sensor. The corresponding noise values for the FPIX0 p-spray sensor was $80 \pm 10 \mathrm{e}^{-}$. In addition, we found an equivalent charge noise due to the external buffer amplifier and ADC of $400 \pm 150 \mathrm{e}^{-}$for FPIX0 p-stop. The corresponding external noise values for the FPIX0 p-spray sensor were $185 \pm 20 \mathrm{e}^{-}$. The FPIX1 chips have four threshold inputs (one for each comparator in the 2-bit FADC implemented in every cell). We found a set of four average threshold values in nominal running conditions, for the FPIX1 p-stop, of about $3780 e^{-}, 4490 e^{-}, 10290 e^{-}$, and $14680 e^{-}$, with a spread of about $380 e^{-}$. The amplifier noise was measured to be $110 \pm 30 e^{-}$. The relatively high FPIX1 readout threshold in the test beam was due to noise and pickup problems in a printed circuit board interface. An FPIX1 test module, with up to 5 chips bump bonded to an ATLAS tile-1 sensor, has been operated stably in bench tests with discriminator threshold set below $1500 \mathrm{e}^{-}$.

\section{Results}

\subsection{Charge collection}

Charge collection can be studied in detail for the FPIX0-instrumented sensors, thanks to the 8-bit analog information and the absolute calibration. Figure 3 shows that the p-spray sensor suffers sizeable charge collection inefficiency between columns, especially on the column boundaries which include the "punch-through biasing" network円. Our measurement of this charge loss is consistent with previous measurements made by the ATLAS pixel collaboration[6]. The measured pulse height distributions were fit using a Landau function convoluted with a Gaussian [7]. Figure 4 shows the pulse height distributions for the FPIX0instrumented p-stop sensor. The "improved Landau" function fits the experimental data quite well, except the bump at $\sim 50000 \mathrm{e}^{-}$which is due to saturation of the off-chip buffer amplifier/ADC combination. In addition, about $0.7 \%$ of the events have

1 These charge losses are thought not to be intrinsic to the p-spray technology, but a feature of this particular sensor design, where each biased $\mathrm{n}^{+}$implant pixel is surrounded by a floating $\mathrm{n}^{+}$implant ring. The charge collection inefficiency is believed to be mostly due to the presence of this ring[6]. 


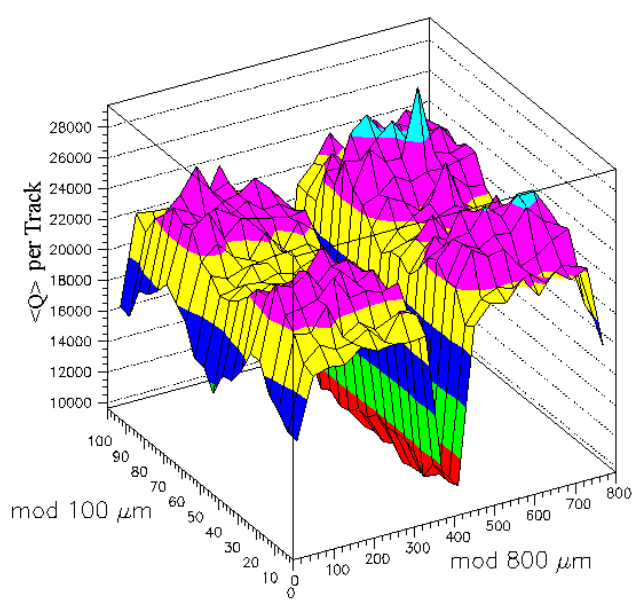

Fig. 3. The average pulse height versus track position for the CiS p-spray sensor bump-bonded to an FPIX0.

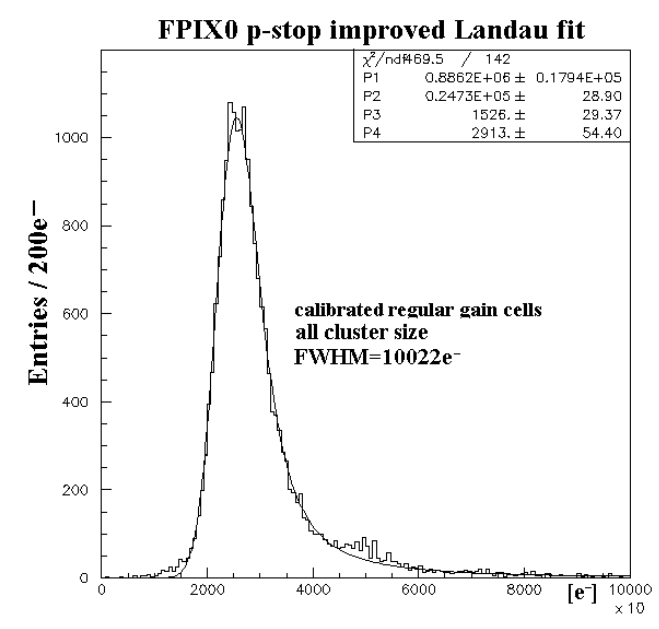

Fig. 4. Pulse height distribution for the CiS p-stop sensor bump-bonded to an FPIX0.

a charge collected in the FPIX0 pstop sensor less than $15000 \mathrm{e}^{-}$(for these values the Landau distribution predicts a very small probability), indicating that the p-stop sensor also suffers a very small charge collection inefficiency. Studies show that this inefficiency is concentrated at the four corners of the sensor pixels.

We find that the charge collected by the CiS p-spray sensor is about $24 \%$ less than the charge collected by the p-stop sensor. The most probable and average charge collected are respectively $20000 \pm 70 \mathrm{e}^{-}$and $23100 \pm 70 \mathrm{e}^{-}$for the CiS p-spray sensor (considering only tracks far away from the inter-pixel boundary). For the CiS p-stop sensor, the most probable charge collected is $24730 \pm 30 \mathrm{e}^{-}$, and the average charge collected is $30100 \pm 30 \mathrm{e}^{-}$.

\subsection{Spatial Resolution}

The tracks used to study pixel spatial resolutions were fit using data from the SSD telescope and from pixel detectors other than the device under test, using a Kalman-filter. The coordinate measured by a pixel detector is obtained by the position of the center of the cluster of hit pixels associated with a track, plus a correction (conventionally called the $\eta$ function) which is a function of the charge sharing, the cluster width, and the track angle. For this analysis, we have used a linear "head-tail" algorithm for computing the $\eta$ function, which ignores the charge deposited in pixels in the interior of a cluster, and uses only the charge deposited on the edges of the cluster[8]. Two specific examples of residual distributions are shown in Figure 5 with the Gaussian fits superimposed. Clearly, the residual distributions are not Gaussian, especially at zero degrees where for a significant fraction of the time only one pixel is hit. The residual distributions also have more entries far from zero than the Gaussian fits. This can be clearly seen in the data taken at ten degrees, when 

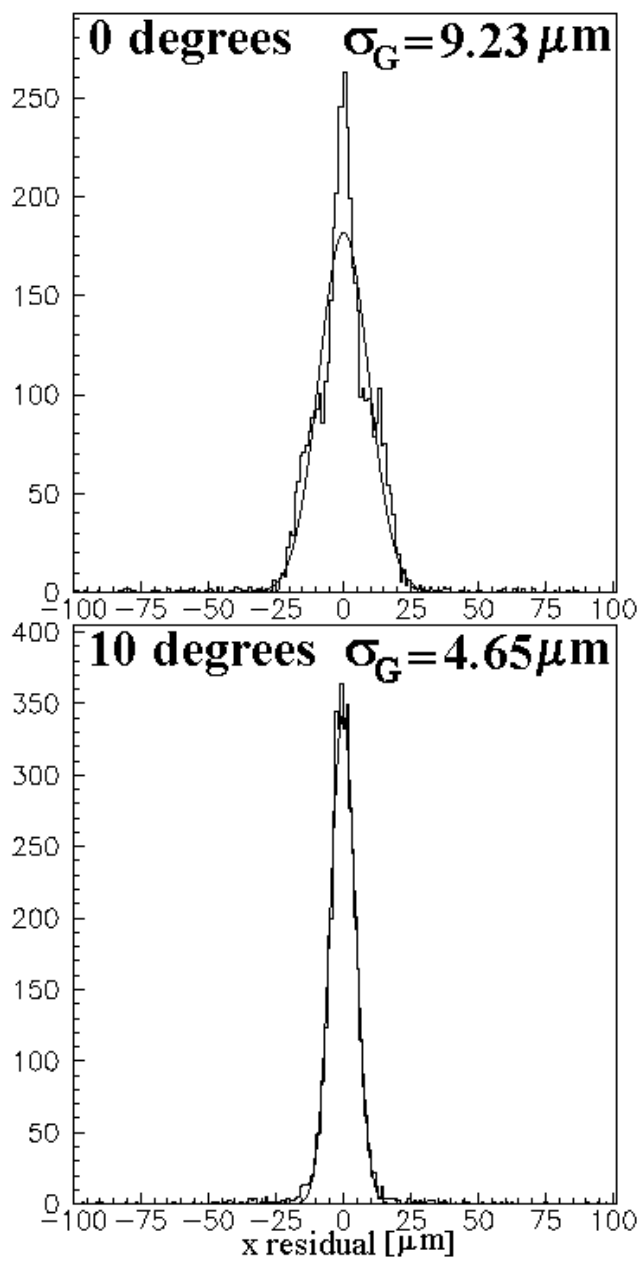

Fig. 5. Residual distributions for the FPIX0 p-stop detector for data taken with $\mathrm{V}_{\text {bias }}=-140 \mathrm{~V}, \mathrm{Q}_{\text {th }}=2500 \mathrm{e}^{-}$and two different track inclinations.

there is always charge sharing. The origin of these "tails" is attributed to the emission of $\delta$-rays which skew the charge sharing and degrade the resolution. Nonetheless, the Gaussian standard deviations provide a reasonably good characterization of the width of the central peak for both plots.

The residual distribution widths, obtained for several track angles and different detectors, are shown in Figure 6 . The experimental results are in good agreement with the simula-

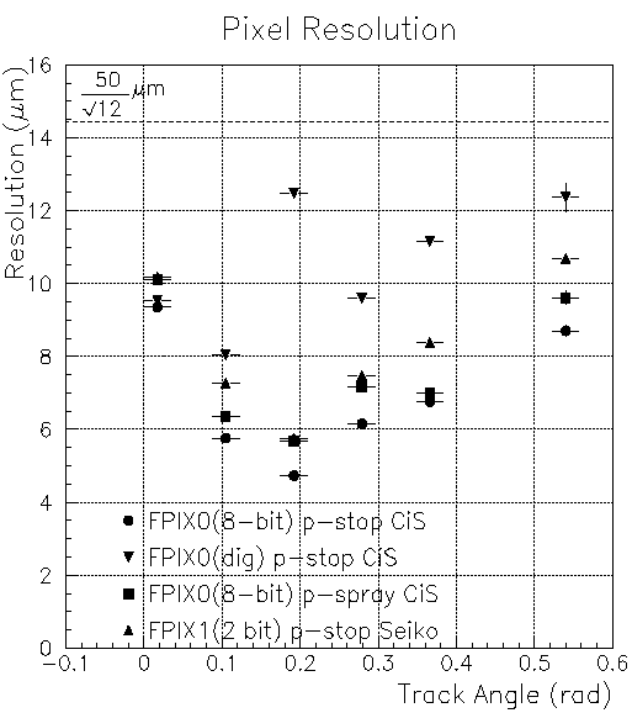

Fig. 6. Position resolution along the short pixel dimension as a function of beam incidence angle for several detectors.

tion results described in [9]. We have also computed residual distributions for this data set without using any charge sharing information. These "digital" resolution results are included in Figure 6. The resolution for the FPIX1-instrumented p-stop detector is slightly worse than the results that we obtained by degrading by software the FPIX0-instrumented p-stop pulse height information to 2-bit equivalents. This is because the main effect degrading the resolution is the high threshold and the 2-bit analog information has only a minor effect. In fact, the FPIX1instrumented detector was operated with a discriminator threshold of $\sim 3780 \mathrm{e}^{-}$, while the FPIX0instrumented detector was operated with a discriminator threshold of $\sim 2500 \mathrm{e}^{-}$. Moreover, the results obtained for a p-spray detector with a threshold of $\sim 2200 \mathrm{e}^{-}$show the extent to which the charge losses in the p-spray sensor degrade the spatial 

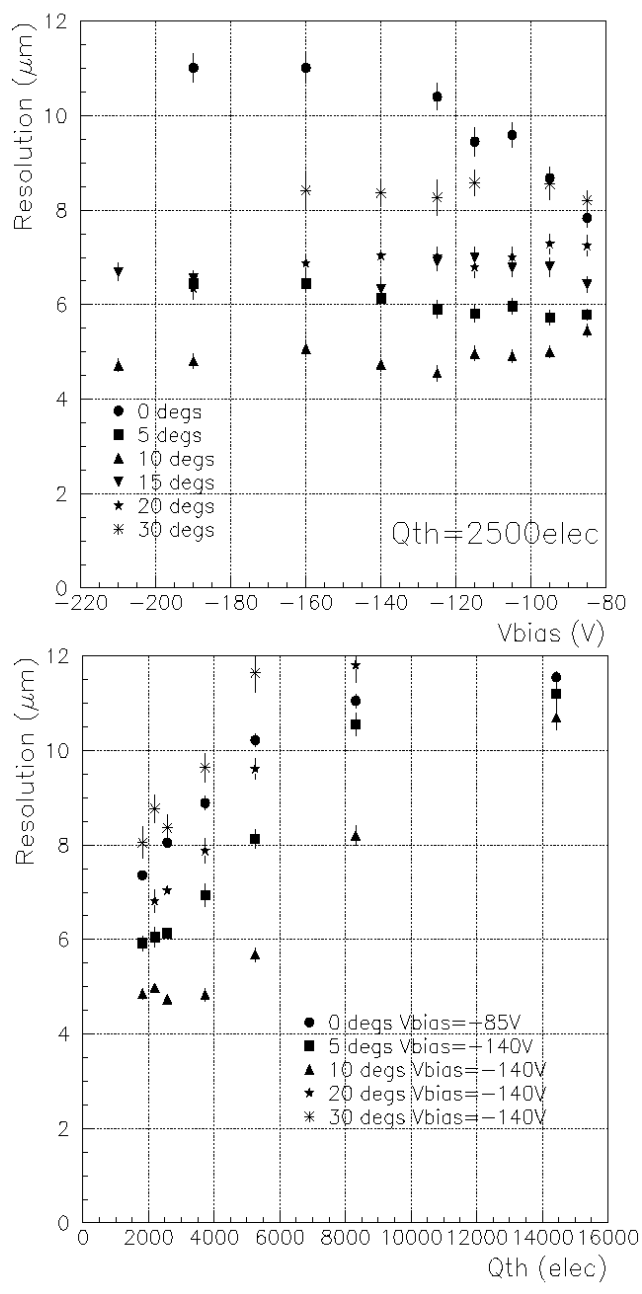

Fig. 7. Spatial resolution versus bias voltage (upper plot) and versus readout threshold (lower plot). The data are from FPIX0-instrumented p-stop sensor with a depletion voltage of $85 \mathrm{~V}$.

resolution.

Figure 7 shows how the position resolution is affected by changes in the sensor bias voltage and the discriminator threshold. The relative sensitivity of the pixel sensor position resolution to these parameters is very important. In fact, the variation of the bias and effective threshold are similar to what is expected when the radiation damage influences the sensor bulk properties and the charge collection efficiency. The data show, for large track angles, not too much sensitivity to the bias voltage, because the charge-sharing is dominated by the track inclination. At small track angle, when the diffusion gives a substantial contribution to the charge-sharing, the sensor bias is important. The effect of the readout threshold is always significant, but the spatial resolution is still better than $10 \mu \mathrm{m}$ up to a threshold of $4000 e^{-}$.

\subsection{Resolution function shape}

The pixel residual distribution (or resolution function) deviates from a Gaussian in two important ways. First, when tracks pass through one pixel only the residual distributions are well fitted by a square function convoluted with a Gaussian. Second, when tracks pass through more pixels we have found that our experimental residual distributions can be fitted by the sum of a Gaussian term $F_{G}$ and a term $F_{N G}$ which is a square with edges that decrease like a power of $1 / \mathrm{x}$ :

$$
F_{N G}(x)=\left\{\begin{array}{l}
\frac{A_{p l}}{\left|r_{c}\right|^{\gamma}} \text { for }|x|<r_{c} \\
\frac{A_{p l}}{|x|^{\gamma}} \text { for }|x|>r_{c}
\end{array}\right.
$$

where $A_{p l}$ is a normalization constant, $r_{c}$ is the half width of the constant term, and $\gamma$ is the exponent of the power law. Figure 8 shows the 


\section{Summary}

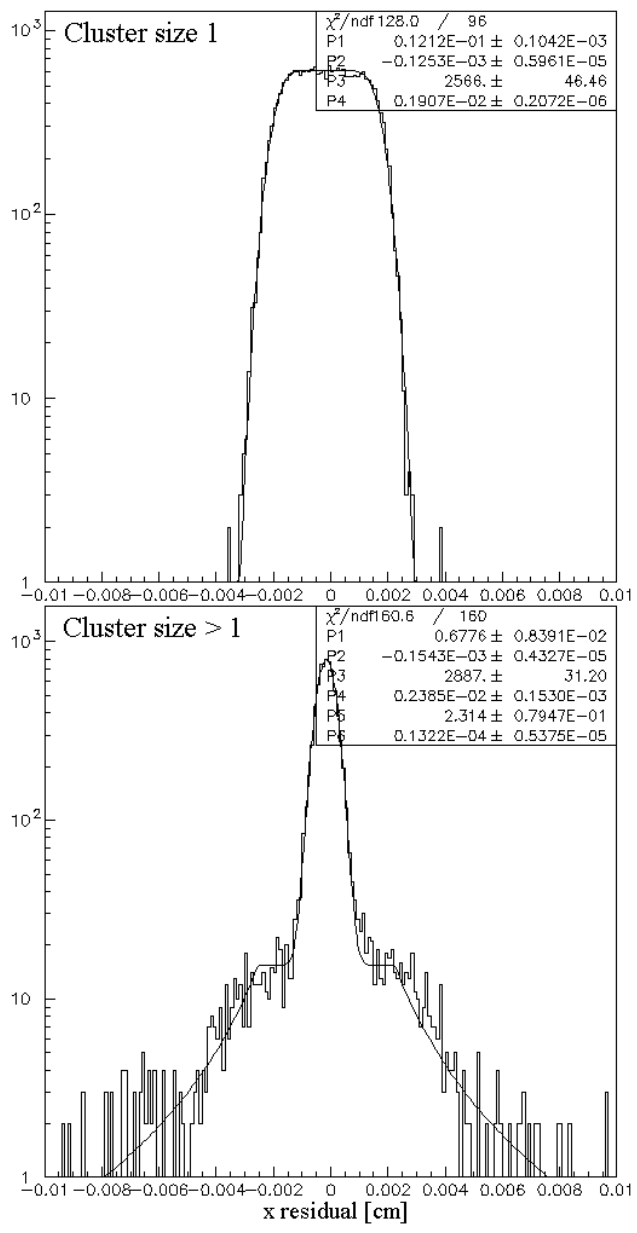

Fig. 8. Experimental residual distributions fitted as described in sec. 3.3.

experimental resolution functions in log scale taken with the beam nominally at normal incidence for cluster size one and cluster size bigger than one. In this last case, we find a satisfactory representation of the data using eq. 1 with the following set of parameters: $\gamma=2.3, r_{c}=23.8 \mu \mathrm{m}$, and $A_{p l}$ set so that $F_{N G}$ accounts for about $18 \%$ of the total number of entries in the distribution.
We have described the results of the $\mathrm{BTeV}$ silicon pixel detector beam test. The pixel detectors under test used samples of the first two generations of Fermilab pixel readout chips, FPIX0 and FPIX1, (indium bump-bonded to ATLAS sensor prototypes). The spatial resolution achieved using analog charge information is excellent for a large range of track inclination. The resolution is still very good using only 2-bit charge information. A relatively small dependence of the resolution on bias voltage is observed. The resolution is observed to depend dramatically on the discriminator threshold, and it deteriorates rapidly for threshold above $4000 e^{-}$.

\section{References}

[1] C. Newsom, Overview of the BTeV Pixel Detector, see these proceedings.

[2] T. Rohe, et al., Nucl. Instr. and Meth. A409 (1998) 224.

[3] T. Zimmerman, et al., IEEE Trans. Nucl. Sci. Vol.40 No.4 (1993) 736.

[4] S. Zimmerman, et al., IEEE Trans. Nucl. Sci. Vol.43 No.3 (1996) 1170.

[5] D.C. Christian, et al., Nucl. Instr. and Meth. A 435 (1999) 144.

[6] F. Ragusa, Nucl. Instr. and Meth. A 447 (2000) 184.

[7] S. Hancock, et al., Nucl. Instr. and Meth. B1:16 (1984) 16. 
[8] R. Turchetta, Nucl. Instr. and Meth. A 335 (1993) 44.

[9] M. Artuso, Spatial resolution predicted for the BTeV pixel sensor, see these proceedings. 